\title{
Coencapsulation of tumor lysate and CpG-ODN in PLGA-microspheres enables successful immunotherapy of prostate carcinoma in TRAMP mice
}

\author{
Marc Mueller ${ }^{\mathrm{a}, *}$, Wilfried Reichardt ${ }^{\mathrm{b}}$, Julia Koerner ${ }^{\mathrm{a}}$, Marcus Groettrup ${ }^{\mathrm{a}, \mathrm{c}, *}$ \\ a Division of Immunology, Department of Biology, University of Konstanz, D-78457 Konstanz, Germany \\ ${ }^{\mathrm{b}}$ Medical Physics, Department of Radiology, University Hospital Freiburg, D-79106 Freiburg, Germany \\ c Biotechnology Institute Thurgau at the University of Konstanz, 8280 Kreuzlingen, Switzerland
}

\begin{abstract}
A B S T R A C T
Biodegradable poly(lactide co glycolide) (PLGA) microspheres (MS) deliver antigens and toll like receptor (TLR) ligands to antigen presenting cells (APC) in vitro and in vivo. PLGA MS microencapsulated model antigens are efficiently presented on MHC class I and II molecules of dendritic cells and stimulate strong cy totoxic and $\mathrm{T}$ helper cell responses enabling the eradication of pre existing model tumors. The application of tumor lysates as a source of antigen for immunotherapy has so far not been very successful also due to a lack of suitable delivery systems. In this study we used PLGA MS with co encapsulated tumor lysates and CpG oligodeoxynucleotides ( $\mathrm{CpG}$ ODN) as well as microencapsulated polyl:C in order to elicit anti tumor re sponses. Immunization of mice with such mixtures of MS yielded substantial cytotoxic T cell (CTL) responses and interfered with tumor growth in TRAMP mice, a pre clinical transgenic mouse model of prostate carcino ma, which has previously resisted dendritic cell based therapy. As an important step towards clinical appli cation of PLGA MS, we could show that $\gamma$ irradiation of PLGA MS sterilized the MS, without reducing their efficacy in eliciting CTL and anti tumor responses in subcutaneous tumor grafts. Since PLGA is approved for clinical application, sterilized PLGA MS containing tumor lysates and TLR ligands hold promise as anti tumor vaccines against prostate carcinoma in humans.
\end{abstract}

\section{Introduction}

Prostate cancer (PCa) is the second leading cause of cancer related death and the most frequently diagnosed cancer among men in western industrialized countries. In about $80 \%$ of all men above 70 years of age at least latent prostate tumors could be detected [1]. The survival rate lies in the range of $8090 \%$ if the tumor at the time of diagnosis is limited to the prostate gland itself, but only $30 \%$ of patients survive with metastat ic tumors. Diagnosis of prostate cancer is reliant on the Gleason score, prostate specific antigen (PSA) levels or the level of early prostate can cer antigen 2 (EPCA 2). Modern standard therapy for tumors of the prostate includes radical surgery, radiotherapy or hormone ablation therapy. In most cases, hormone therapy decelerates progression or sta bilizes metastatic disease for several months or years. Unfortunately, in cidences of hormone resistance accumulate during extended therapy.

In the past two decades, immunotherapy has been intensively inves tigated as a novel option to treat hormone refractory, metastasizing PCa [2]. Since primary tumor is often surgically removed with curative in tent, the tissue can be cryopreserved and used for immunotherapy if a

\footnotetext{
* Corresponding authors at: Lehrstuhl Immunologie, Universität Konstanz, Universitätsstrasse 10, D-78457 Konstanz, Germany.

E-mail addresses: marc.mueller@uni-konstanz.de (M. Mueller), Marcus.Groettrup@uni-konstanz.de (M. Groettrup).
}

relapse should occur later on. An approach using lysate of melanoma tu mors was pioneered in 1988 by Mitchell and colleagues [3]. The tumor lysates were applied together with DETOX, a water in oil emulsion con taining immunostimulatory adjuvants derived from bacteria. The vac cine Melacine ${ }^{\circledR}$ was launched by Corixa Corporation with a significant increase in relapse free survival in melanoma patients in first trials. Later studies revealed an influence of the HLA type of the patients on the probability of therapeutic success [4] and confirmed the low but consistent efficacy in clinical phase I and II trails [5]. The carrier system employed in this vaccine is similar to incomplete Freund's adjuvans (IFA) [6], which is still used in experimental immunotherapy of melano ma patients [7], but will most likely never be approved for standard clinical use, due to adverse side effects.

The approach to deliver PCa tumor lysate pursued in this study re lies on co encapsulation of the lysates in biodegradable poly(D,L lactide co glycolide) microspheres (PLGA MS). The PLGA MS system compares favorably with IFA and has been proven to be effective in vitro and in immunotherapy of syngeneic model tumors in mice [8]. Antigens and TLR ligands microencapsulated in PLGA MS are taken up by human and murine dendritic cells and macrophages very effi ciently in vitro and in vivo without negatively influencing cardinal properties like migratory capacity, cytokine secretion or antigen pre sentation [9]. The co encapsulation of protein or peptide antigens and TLR ligands into PLGA MS protects them from degradation and 
provides a long lasting depot for sustained and prolonged immune re sponses [10 12]. The particle size of PLGA MS obtained by spray drying is about $110 \mu \mathrm{m}$, which facilitates the uptake of antigens by APCs [13], followed by transport to the secondary lymphoid organs [14]. We and others could show that the co encapsulation of the model antigen ovalbumin and $\mathrm{CpG}$ oligodesoxynucleotides (ODN) yields substantial cytotoxic T lymphocyte (CTL) responses as well as striking anti tumor responses $[15,16]$. These are excellent prerequi sites for efficient in vivo loading of professional antigen presenting cells (APC) and priming of CTL mediated anti tumor responses. In 2007 it was shown, that encapsulation of tumor cell lysate and subse quent injection leads to delayed tumor occurrence, even without the usage of any immune stimulatory adjuvant [17]. Other laboratories could show that encapsulation of endogenous antigens can break tol erance [18] and that co encapsulated tumor lysates with CpG ODN led to impaired tumor growth in mice [19]. We could further improve this system by adding a second Toll like receptor (TLR) ligand, sepa rately encapsulated. This has been shown to influence DCs in terms of $\mathrm{T}$ helper (Th) cell polarization [20]. Furthermore, the frequently used spray drying technique is a rapid, highly reproducible and easy to scale up process to encapsulate tumor lysate from resected PCa in combination with two TLR ligands.

The best characterized mouse model to test immunotherapy of $\mathrm{PCa}$ is the transgenic adenocarcinoma mouse prostate (TRAMP) model. TRAMP mice are transgenic for the SV40 large T antigen expressed after puberty under the control of the rat probasin regula tory elements [21]. Male TRAMP mice remain healthy until puberty (weeks 4 to 5). In the weeks thereafter TRAMP mice progressively de velop prostate intraepithelial neoplasia, with documented progres sion to invasive carcinoma of epithelial origin, closely resembling the pathology of human PCa [22] and subsequent metastasis forma tion [23].

Attempts to limit or abrogate tumor growth in male TRAMP mice with immunotherapy have been met with limited success. In particular, cellular immunotherapy with dendritic cells failed to elicit significant cytolytic responses when applied at the age of 9 weeks i.e. after the onset of tumor growth which was most likely due to the T cell tolerizing capacity of TRAMP tumors [24 26]. In this study, we show that immunization of male TRAMP mice with PLGA MS containing TRAMP prostate tumor lysate (PTL) and CpG oligonucleotides and PLGA MS containing polyI: $\mathrm{C}$ at the age of 10 weeks is able to prevent or strongly reduce the growth of PCa in TRAMP mice. Given that PLGA is a material approved for clinical application [27] and that PCa tissue from patients is often avail able, this approach holds promise to be tested in the clinical setting.

\section{Materials and methods}

\subsection{Preparation of MS}

MS were prepared from 14 kDa PLGA 50:50 carrying hydroxyl and carboxyl end groups (Resomer RG502H, Boehringer Ingelheim, Ingelheim, Germany). The lysates and TLR ligands were microencapsulated by spray drying. In the present study, $1 \mathrm{ml}$ (15 mg protein / $\mathrm{ml}$ ) of lysed TrampC2 cells [28], stably transfected with full length cytosolic ovalbumin (Vf10L; experiments of Fig. 1), or ovalbumin expressing EG.7 thymoma cells (EG.7L; experiments of Fig. 2), or prostate tumors resected from TRAMP mice (PTL; experiments of Figs. 3 and 4) were co encapsulated with $5 \mathrm{mg}$ CpG oligodeoxynucleotides (with phosphothioate backbone; CpG ODN 1826, Microsynth, Balgach, Switzerland) dissolved in $0.25 \mathrm{ml} 0.1 \mathrm{M}$ $\mathrm{NaHCO}_{3}(\mathrm{MSVf} 10 \mathrm{~L} / \mathrm{CpG}$ or MSEG.7L/CpG). One PLGA MS preparation con tained only $0.5 \mathrm{mg}$ polyl:C (Calbiochem, VWR, Dietikon, Switzerland) dis solved in $0.5 \mathrm{ml} 0.1 \mathrm{M} \mathrm{NaHCO}_{3}$ (MS polyl:C) and another contained $50 \mathrm{mg}$ ovalbumin (Grade V, Sigma) co encapsulated with $5 \mathrm{mg}$ CpG (MS Ova/ $\mathrm{CpG}$ ). The respective aqueous phases were mixed with $1 \mathrm{~g}$ of PLGA dissolved in $20 \mathrm{ml}$ of dichloromethane (organic phase). The two phases were subsequently emulsified by ultrasonication (Hielscher, UP200 H, Ampl. 40\%) for $10 \mathrm{~s}$ on ice. The w/o dispersion was immediately spray dried (Büchi, Mini Spray Dryer 191) at a flow rate of $2 \mathrm{ml} / \mathrm{min}$ and inlet/ outlet temperatures of $40 / 37^{\circ} \mathrm{C}$. The MS were washed out of the spray dryer's cyclone with $0.05 \%$ Synperonic (Synperonic $® F 68$, Serva Electrophoresis, Heidelberg, Germany), collected on a cellulose acetate membrane filter and dried under reduced pressure ( $20 \mathrm{mbar}$ ) for $18 \mathrm{~h}$ at room temperature. PLGA MS were stored at $4{ }^{\circ} \mathrm{C}$. PLGA MS were $\gamma$ irradiated in glass vials with a $\mathrm{Co}^{60}$ derived dose of $25 \mathrm{kGy}$ for $4 \mathrm{~h}$, using a GS3000 $\gamma$ irradiation facility of Synergy Health Radeberg (Radeberg, Germany). Immediately before use, MS were dispersed in PBS by ultrasonication for $30 \mathrm{~s}$. The sterility of the irradiated micro spheres was confirmed by incubating them in tissue culture media at $37^{\circ} \mathrm{C}$ for three days during which no growth of bacteria or fungi was recorded in contrast to non irradiated controls.
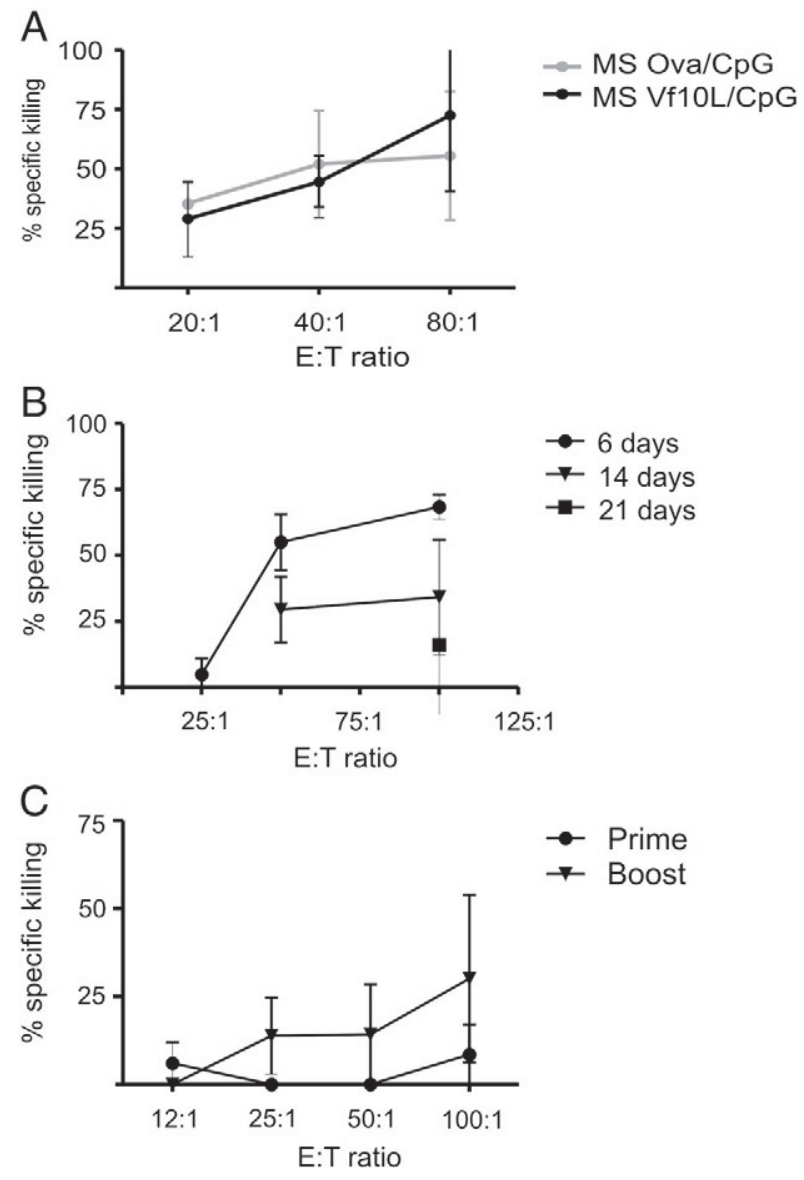

Fig. 1. Ex vivo DELFIA $®$ EuTDA cytotoxicity assay of mouse splenocytes after immunization with Vf10 PCa tumor lysate containing PLGA-MS. (A) C57BL/6 mice $(n=2)$ were immunized with $5 \mathrm{mg}$ MS-polyl:C and either $5 \mathrm{mg}$ MS-Vf10-Lysate/CpG (MS$\mathrm{Vf10L/CpG)}$ or with $5 \mathrm{mg} \mathrm{MS-Ova/CpG} \mathrm{(MS-Ova/CpG);} \mathrm{Vf10} \mathrm{cells} \mathrm{are} \mathrm{ovalbumin} \mathrm{tran-}$ sfectants of the PCa line TrampC2. On day 6 after immunization, splenocytes were monitored for ex vivo cytotoxicity against Vf10 target cells; no statistically significant difference was recorded between both data sets. (B) C57BL/6 mice $(n=6)$ were immunized with 5 mg MS-polyl:C and 5 mg MS-Vf10-Lysate/CpG. On day 6 (circles), day 14 (triangles), and day 21 (square) after immunization, splenocytes were monitored for ex vivo cytotoxicity against Vf10 cells. (C) Male TRAMP mice $(n=4)$ were immunized at the age of 10 weeks twice with $5 \mathrm{mg}$ MS-polyl:C and $5 \mathrm{mg}$ MS-Vf10-Lysate/CpG in an interval of four weeks. Six days after each immunization blood lymphocytes were analysed for ex vivo cytotoxicity. Values are shown for the prime (squares) and for the boost (triangles) at different effector to target ratios in \% specific killing of $5 \times 10^{4}$ Vf10 target cells. The graphs show representatives of three independent experiments ( $A$ and $B$ ) or a summary of two independent experiments with the same outcome (C). 


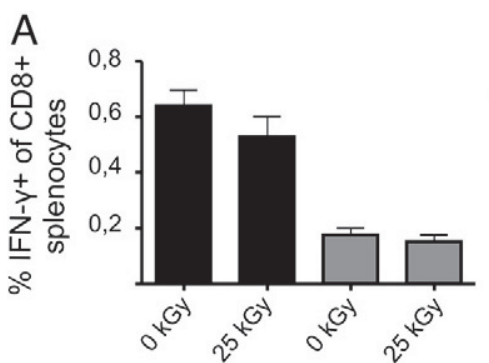

B

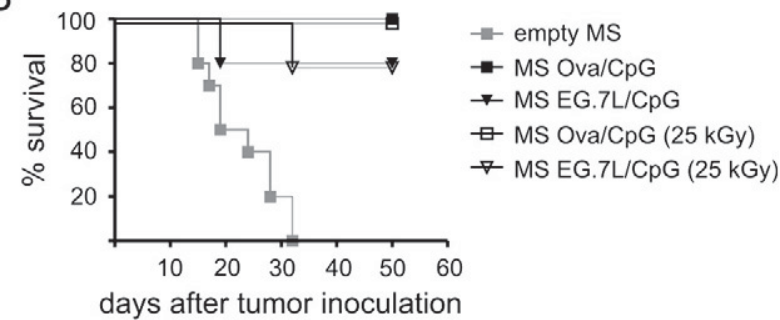

Fig. 2. Sterilization of PLGA-MS by $\gamma$-irradiation does not alter the success of immunotherapy. (A) C57BL/6 mice $(n=3)$ were immunized with $5 \mathrm{mg}$ MS containing Ova/CpG or $5 \mathrm{mg}$ MS-EG.7-Lysate/CpG in combination with $5 \mathrm{mg}$ MS-polyI:C, either $\gamma$-irradiated with a dosage of $25 \mathrm{kGy}$ ( $25 \mathrm{kGy}$ ) or not sterilized ( $0 \mathrm{kGy}$ ). Six days after immunization, an ICS was performed to monitor success of the immunization. Values are given in $\% \mathrm{CD}^{+} / \mathrm{IFN}-\gamma^{+}$ lymphocytes, \pm SEM. The experiment was conducted twice, with similar results. (B) C57BL/6 mice (at least 5 per group) were challenged with $5 \times 10^{5}$ EG-7 cells s.c. at the right flank. As soon as palpable tumors were noted, mice were immunized with $5 \mathrm{mg}$ MSpolyl:C and either $5 \mathrm{mg}$ MS-EG-7Lysate/CpG, which had been $\gamma$-irradiated (open triangles) or not irradiated (filled triangles), or with $5 \mathrm{mg} \mathrm{MS}-\mathrm{Ova} / \mathrm{CpG}$, which had been $\gamma$-irradiated (open squares) or not irradiated (filled squares). The control group was immunized with $5 \mathrm{mg}$ empty MS (grey squares). Mice were sacrificed when the mean tumor size, assed by two orthogonal measurements, exceeded a size of $15 \mathrm{~mm}$. Values are given in \% survival. The graph represents one out of three independent experiments with similar outcomes.

\subsection{Particle size measurement}

Approximately $10 \mathrm{mg}$ of PLGA microparticles were placed in a small agate mortar and dispersed with a agate pistil in a small drop of polysorbate 20 (Fluka); then, $2 \mathrm{ml}$ of water were added to make a homogenous slurry, which was sonicated for 2 times 15 seconds. Particle sizes were measured by laser light scattering (Mastersizer 2000, version 5.60, Malvern Instruments, Malvern, UK). Each sample was measured twice with in between ultrasonication of the sample slurry inside the sample dispersion unit.

\subsection{Zeta potential measurement}

The surface charge of the microparticles was determined by zeta potential measurement using Delsa Nano C (Beckman Coulter). For that purpose, approximately $10 \mathrm{mg}$ microparticles were wetted with approx. $10 \mu \mathrm{l}$ of polysorbate 20 using a mortar and pistil. Then, the particles were dispersed by adding approximately $5 \mathrm{ml}$ of purified and sterile filtered water. The slurry had a $\mathrm{pH}$ value of 6.5 . The reported zeta potential value is the average of three measurements.

\subsection{Total protein content and release kinetics of PLGA MS}

$10 \mathrm{mg}$ of PLGA MS were either dissolved in $500 \mu \mathrm{l}$ DMSO (con tent) or incubated at $37{ }^{\circ} \mathrm{C}$ in a volume of $250 \mu \mathrm{l}$ PBS (release kinet ics). At time points of $1 \mathrm{~h}, 8 \mathrm{~h}, 1,2,3,4,7,8,9,10,11,14,21$, and 28 days, respective samples were spun down for $2 \mathrm{~min}$ at $5000 \mathrm{rpm}$ and supernatant was collected and stored at $-20^{\circ} \mathrm{C}$. For BCA protein assay (Pierce, Thermo Fisher Scientific.). Samples of DMSO solution were TCA precipitated and resuspended in PBS.

\subsection{SDS PAGE and Western blot analyses}

For analysis by denaturing SDS PAGE, samples were boiled in SDS sample buffer with $10 \% \beta$ mercaptoethanol. The proteins were either detected on $12 \%$ acrylamide gels by Coomassie staining or transferred onto a nitrocellulose membrane for Western blot analysis, using a Biometra FastBlot B34 (Biometra, Germany) semi dry blotting device. Antibodies for PAP and PSA were applied over night at $4{ }^{\circ} \mathrm{C}$ after blocking the membrane with $3 \% \mathrm{BSA}$ for $2 \mathrm{~h}$ at room temperature. HRP conjugated secondary antibodies were used for $2 \mathrm{~h}$ at room tem perature prior to detection using a ChemiDoc imaging system (BioRad, Germany).

\subsection{Mice and immunizations}

C57BL/6 mice and mice, transgenic for adenocarcinoma of the mouse prostate (TRAMP) [21], were originally purchased from Jackson Laboratories. All mice were kept in a specific pathogen free facility and used at 810 weeks of age, if not described differ ently. Animal experiments were approved by the review board of Regierungspräsidium Freiburg. PLGA MS were injected s.c. at the base of the tail.

\subsection{Cell lines and media}

The ovalbumin expressing EL 4 thymoma transfectant EG 7 was kindly provided by Dr. Wolfram Osen (DKFZ Heidelberg, Germany) and kept in RPMI medium. Media were purchased from Invitrogen Life Technologies and contained GlutaMAX, 10\% fetal calf serum, and $100 \mathrm{U} / \mathrm{ml}$ of penicillin/streptomycin $(\mathrm{P} / \mathrm{S})$. For the clone Vf10, prostate tumor derived TrampC2 cells [28] were transfected with a plasmid encoding for full length cytosolic ovalbumin [29]. Vf10 cells were kept in DMEM ( pyruvate), complemented with 5\% FCS, 5\% Nu serum, $5 \mu \mathrm{g} / \mathrm{ml}$ Insulin, $10^{-8} \mathrm{M}$ DHT and $1 \% \mathrm{P} / \mathrm{S}(5 \mathrm{ml})$. To main tain the expression of ovalbumin in EG 7 and Vf10 cells, the media contained G418 $(0.2 \mathrm{mg} / \mathrm{ml})$.

\subsection{Intracellular cytokine staining}

For intracellular cytokine staining (ICS), splenocytes were isolated and incubated with or without $10 \mu \mathrm{M}$ SIINFEKL peptide (Eurogentec, Cologne, Germany) in the presence of brefeldin A (10 $\mu \mathrm{g} / \mathrm{ml}$, Sigma Aldrich) for $5 \mathrm{~h}$ at $37^{\circ} \mathrm{C}$. After washing, cells were stained with PE Cy5 conjugated anti mouse $\mathrm{CD} 8 \alpha$ antibody (BD Biosciences) for $20 \mathrm{~min}$ at $4{ }^{\circ} \mathrm{C}$. The cells were washed again and fixed with $4 \%$ para formaldehyde in PBS for 10 min at room temperature. After washing the cells twice with PBS, cells were labeled intracellularly with FITC conjugated rat anti IFN $\gamma$ antibody (clone XGM1.2, diluted in PBS / $0.1 \%$ saponin) at $4{ }^{\circ} \mathrm{C}$ overnight. The next day, cells were washed and resuspended in PBS for flow cytometry. Background levels of each sample (without peptide) were subtracted.

\subsection{ELISPOT analyses}

Commercially available antibody pairs for IFN $\gamma$ (BD Biosciences) or granzyme B (R\&D Systems) were used according to the manufacturer's instructions. Briefly, $1 \times 10^{6}$ splenocytes were co incubated with $1 \times 10^{5}$ MS PTL/CpG loaded DC2.4 in anti IFN $\gamma$ antibody coated $(10 \mu \mathrm{g} / \mathrm{ml})$ ELISPOT 96 well plates which were subsequently blocked. After over night incubation biotinylated antibodies were added $(2 \mu \mathrm{g} / \mathrm{ml})$ for $2 \mathrm{~h}$ of incubation, followed by streptavidin alkaline phosphatase enzyme conjugates for 40 minutes at room temperature. Spots of the dried plates were counted using an ImmunoScan instrument (C.T.L., Cellular Technol ogy Ltd.). 
A

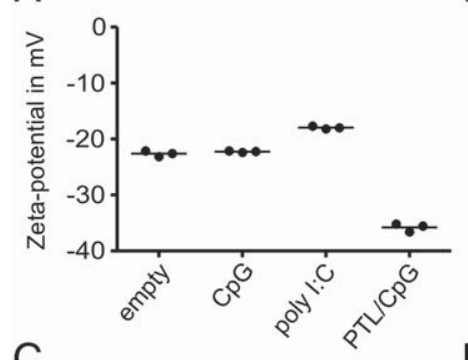

C
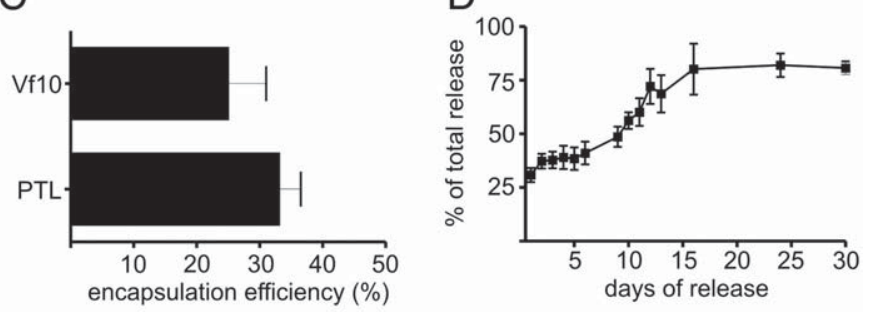

$\mathrm{E}$

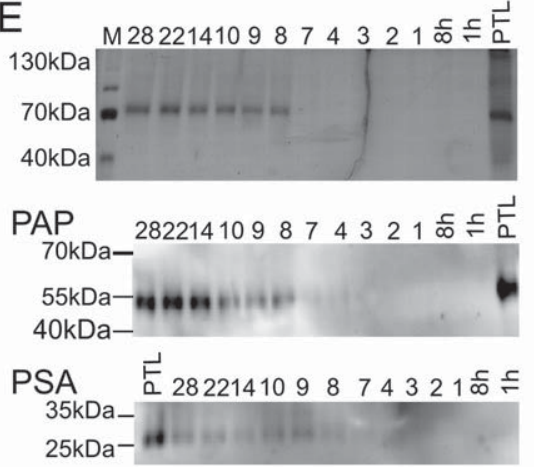

Fig. 3. Characterization of PLGA encapsulation of tumor lysate. (A) Zeta potential of empty MS (empty), of MS containing CpG or poly I:C, and of MS containing prostate tumor lysate (PTL) and CpG (PTL/CpG). (B) Number weighted size distribution (upper graph) and volume weighted size distribution (lower graph) of MS containing PTL and CpG. (C) Encapsulation efficiency of Vf10 and PTL in PLGA-MS. $10 \mathrm{mg}$ of each MS-preparation were dissolved in $1.0 \mathrm{ml}$ of DMSO, and protein content was measured by BCA assay. Assessed amounts were compared to theoretical maximal loading and given in \% encapsulation efficiency. (D) Release kinetics of MS containing PTL and CpG (MS PTL/CpG) over a period of 4 weeks. $10 \mathrm{mg}$ of MS were incubated at $37^{\circ} \mathrm{C}$ in a volume of $250 \mu \mathrm{PBS}$. Values are given as \% of the experimentally determined loading. The graph represents a summary of 3 independent experiments with similar outcome. (E) SDS-PAGE with Coomassie staining of cumulative release test samples of different time points (plain numbers repreent days) showing total protein (PTL) (upper image), prostate acidic phosphatase (PAP) specific immunoblot (middle blot), and prostate specific antigen (PSA) specific immunoblot (lower blot).

\subsection{Ex vivo cytotoxicity assay}

For measurement of ex vivo cytolytic activity, a time resolved fluo rometric assay was used. At the indicated time points, splenocytes were isolated and used as effectors in a primary cytotoxicity assays as previously described [30] using DELFIA® EuTDA cytotoxicity re agents (PerkinElmer). In this assay cells are labeled with a cell mem brane permeable reagent Bis(acetoxymethyl) $2,2^{\prime}: 6^{\prime}, 2^{\prime \prime}$ terpyridine $6,6^{\prime \prime}$ dicarboxylic acid (BaTDA). Within the cell the ester bonds are hydrolyzed to form a hydrophilic ligand (TDA) which no longer pas ses the membrane. After cytolysis the ligand is released and incubated with a Europium $\left(\mathrm{Eu}^{3+}\right)$ solution. The Europium and the ligand form a highly fluorescent and stable chelate (EuTDA). The measured signal correlates directly with the extent of cell lysis.

Briefly, $5 \times 10^{4}$ Vf10 cells served as target cells and were labeled with BaTDA. After 4 h of coincubation, supernatants were mixed with Europium solution for $1 \mathrm{~h}$ and subsequently measured at excitation/emission wave lengths of 340/615 nm and a lag time of $200 \mathrm{~ns}$. Specific cytolysis was cal culated as follows: [(counts for immunized mouse - counts for naïve mouse $) \times 100)] /$ (total lyses).
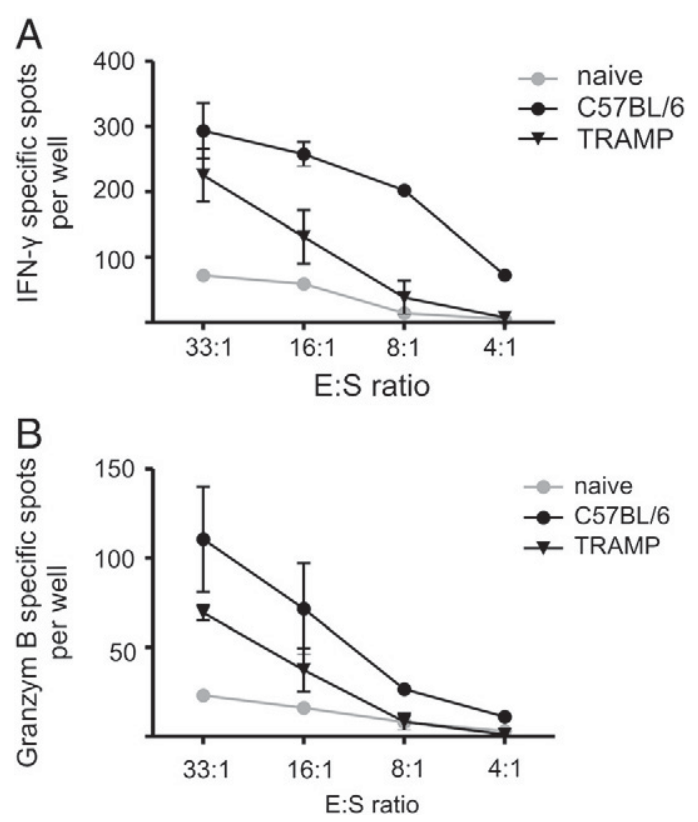

Fig. 4. ELISPOT assay for IFN- $\gamma$ and granzyme B comparing cytotoxic T cell responses in male C57BL/6 and TRAMP mice after immunization with tumor lysate containing PLGA-MS. Male TRAMP mice (triangles, $n=3$ ) or C57BL/6 mice (circles; $n=2$ ), at the age of ten weeks, were immunized with $5 \mathrm{mg}$ MS containing TRAMP prostate tumor lysate co-encapsulated with $\mathrm{CpG}(\mathrm{MS} \mathrm{PTL} / \mathrm{CpG})$ and $5 \mathrm{mg}$ MS polyI:C. Immunizations were repeated three more times at intervals of two weeks. Six days after the last immunization, splenocytes were analyzed by ELISPOT assays for IFN- $\gamma$ and granzyme B, using MS PTL/CpG pulsed DC2.4 as stimulators. Naive C57BL/6 mice (gray circles) served as controls. The graph represents one out of two experiments with similar outcome.

\subsection{Magnetic resonance imaging (MRI) of TRAMP mice}

Prostate tumor volumes of TRAMP mice were imaged using a 9.4 Tesla small bore animal scanner (BioSpec 94/20, Bruker Biospin, Ettlingen, Ger many) equipped with a cylindrical quadrature birdcage resonator with an inner diameter of $38 \mathrm{~mm}$, specifically designed for whole body mouse imaging. The MRI protocol consisted of a localizer and a T2 weighted spin echo RARE (Rapid Acquisition with Relaxation Enhancement) sequence. The RARE sequence was performed to delineate the prostatic tumors from the surrounding abdominal tissue. The RARE sequence (TR/TEeff/ FA: $3000 \mathrm{~ms} / 36 \mathrm{~ms} / 180^{\circ}$, echo train length: 8 ) featured a field of view (FOV) of $30 \times 30 \mathrm{~mm}^{2}$; a matrix size of $256 \times 256$ pixel $^{2}$, and an in plane res olution of $117 \times 117 \mu \mathrm{m}^{2}$. The slice thickness was $0.5 \mathrm{~mm}$ with no slice spacing to achieve contiguous image sets of the whole volume. The number of slices was adjusted to the measured volume (on average 25 ) to ensure complete coverage of the tumors. The total volume of the tumors was de termined using MRI volumetry. Therefore, the perimeter of the tumour was manually traced on each slice image. The tumor volume was then cal culated by adding all the voxel volumes lying within the boundaries of the region of interest (ROI). Total tumor volumes were calculated from sets of contiguous images by summing products of area measurements and slice thickness using the MIPAV software (National Institutes of Health, Bethes da, MD, USA). Differences between groups were assessed by an unpaired Student's $t$ test with Welch's correction.

\section{Results}

3.1. PLGA MS containing tumor lysates induce long lasting cytotoxic $T$ lymphocyte responses in vivo

Lysates derived from autologous tumor cells represent a potential source of tumor associated antigens (TAA) for immunotherapeutic approaches. In order to investigate the potential of biodegradable PLGA MS containing tumor lysates and TLR ligands for anti tumor 
immunotherapy, we resected prostate tumors from 22 week old TRAMP mice, which were subsequently homogenized and the single cell suspension was lysed via seven freeze thaw cycles. We immu nized C57BL/6 mice with MS containing either ovalbumin and CpG ol igonucleotide (MS Ova/CpG, gray line in Fig. $1 \mathrm{~A}$ ) or MS containing tumor lysate derived from the ovalbumin expressing PCa cell line Vf10 and CpG co injected with MS polyI:C (MS Lysate/CpG, black line in Fig. 1A). This regimen was previously shown to yield optimal cytolytic responses (see supplementary figure S1). Six days after im munization, we performed a DELFIA ${ }^{\circledR}$ EuTDA cytotoxicity assay, using splenocytes derived from the immunized mice as effector cells and Vf10 cells (ovalbumin transfected TrampC2 cell line) as targets. Splenocytes from both groups were potent in killing TrampC2 target cells directly ex vivo in a $4 \mathrm{~h}$ assay. As shown in Fig. 1B, the capacity to kill BaTDA labeled target cells lasted at least for 14 days after im munization. On day 21, (squares) only the highest effector to target ratio showed detectable cytotoxic activity. The group that was immu nized 14 days prior to the assay (triangles) showed substantial killing at effector to target ratios of 100:1 and 50:1, but not at the ratio of 25:1. In Fig. 1C, an initial experiment with male TRAMP mice is shown. TRAMP mice were primed at 10 weeks of age and boosted 4 weeks later with MS containing TrampC2 lysate/CpG in combina tion with MS polyI:C. Six days after priming (circles) and six days after the boost (triangles), blood was drawn and peripheral blood lymphocytes were analyzed in an ex vivo DELFIA® EuTDA cytotoxicity assay. Specific killing of $5 \times 10^{4} \mathrm{Vf10}$ target cells was barely apparent after priming, but well detectable after the boost.

\subsection{Sterilization of PLGA MS by $\gamma$ irradiation does not negatively affect anti cancer immunotherapy}

A prerequisite for the application of particulate vaccines in humans is product sterility, which can be obtained by aseptic processing or terminal product sterilization. We decided to $\gamma$ irradiate PLGA MS in order to test if terminal sterilization would alter their immune stimulatory properties. To characterize the sterilized MS, we immunized C57BL/6 mice $(n=16)$ with $\gamma$ irradiated or non irradiated PLGA MS either containing the model antigen ovalbumin or EG7 tumor cell lysate coencapsulated with $\mathrm{CpG}$, in combination with MS polyl:C. After six days, an ICS for intracellu lar INF $\gamma$ was performed and we could not detect a significant influence of $\gamma$ irradiation on the performance of PLGA MS (Fig. 2A). The same PLGA MS were used for immunotherapy of mice bearing EG7 tumors (Fig. 2B). C57BL/ 6 mice were inoculated with $5 \times 10^{5}$ EG7 cells s.c. in the right flank; as soon as tumors became palpable (approx. 10 days after inoculation) groups of mice ( $n \geq 10)$ were treated with PLGA MS as in $2 \mathrm{~A}$, or with empty MS as a control. Monitoring the mice during a pe riod of 50 days after tumor inoculation, we found that immunized groups, but not the control group, mostly showed a total regression of the pre established tumor irrespective of whether or not the MS had been steril ized by $\gamma$ irradiation.

\subsection{Characterization of PLGA MS containing TRAMP tumor lysate and CpG oligonucleotides}

Further product characteristics of the PLGA MS with encapsulated PTL and CPG were determined in terms of particles zeta potential (Fig. 3A ) and size (Fig. 3B for MS PTL/CpG; see supplementary figure S2 for particle sizes of empty MS, MS CpG, and MS polyI:C). Typical for microspheres produced by spray drying, the size distribution was heterodisperse with micro as well as nanoparticles being present in the preparation but the contribution of microsphere volume and antigen amount contributed by nanoparticles were negligible. Fur thermore, we determined the encapsulation efficiency for Vf10 lysate and PTL (Fig. 3C), which was around 30\% in both cases. Fig. 3D shows the in vitro release kinetics of the PTL proteins, displaying an initial burst within $48 \mathrm{~h}$, where approx. $40 \%$ of the encapsulated proteins were released. Within the testing period of 28 days, approx. $80 \%$ of the encapsulated protein was released. PTL release was further assessed by SDS PAGE, which revealed that the fraction of released PTL was amounted to approx. $25 \%$ of the nominally encapsulated PTL (15 $\mu \mathrm{g} / \mathrm{mg}$ PLGA MS) (upper blot of Fig. 3E). Furthermore, SDS PAGE did not evidence any obvious alteration of the proteins released from the PLGA. Immunoblot analyses of released PCa protein antigens showed the gradual release of prostate acidic phosphatase (PAP, $50 \mathrm{kDa}$ ) and prostate specific antigen (PSA, $34 \mathrm{kDa}$; Fig. 3F, middle and lower blot). The blots not only document the presence of the two major PCa markers in the encapsulated and released lysate, but also that a fraction of $\sim 25 \%$ of the nominal $15 \mu \mathrm{g}$ protein/mg PLGA MS was released within 28 days (PSA density of PTL: nominal $=2000$, released within $\mathrm{d} 28=550$; PAP density of PTL: nominal $=1450$, re leased within $\mathrm{d} 28=400$ ).

3.4. Strong cytotoxic T lymphocyte responses can be elicited in TRAMP mice with PLGA MS containing TRAMP tumor lysate and CPG oligonucleotide

In the experiments shown in Fig. 1C, cytolytic activity could be measured directly ex vivo without any restimulation when TRAMP mice were primed and boosted with MS containing Vf10 lysate/CpG in combination with MS polyI:C. Given that robust cytolytic re sponses could be elicited in this set up, we decided to apply a more stringent prime boost regimen in the TRAMP model. C57BL/6 mice or TRAMP mice were immunized at the age of 10 weeks with TRAMP mouse derived prostate cancer lysate (TPL), coencapsulated into PLGA MS with CpG, and injected in combination with MS polyI:C. Immunization was repeated three more times at intervals of two weeks. Six days after the last immunization, mice were sacrificed and splenocytes were analyzed for the production of INF $\gamma$ (Fig. 4A) and granzyme B (Fig. 4B) in a direct ex vivo ELISPOT assay. In both as says, our protocol revealed substantial direct ex vivo responses in both mouse strains indicating that PLGA MS with encapsulated tumor lysate was appropriate to break tolerance and yielded cytolytic $\mathrm{T}$ cell responses against the syngenic tumor.

\subsection{Direct ex vivo killing of TrampC2 cell line by splenocytes from mice immunized with MS PTL/CpG + MS polyI:C}

To further elucidate the quality of the cytotoxic response and to document the independency of the $T$ cell response from the model antigen ovalbumin, we analyzed the ex vivo cytolytic response against the TrampC2 cell line. Six days after two immunizations at an interval of two weeks, splenocytes were either co incubated with BaTDA la beled TrampC2 cells (upper graph of Fig. 5) or with MC57 fibrosarco ma cells (lower graph of Fig. 5). The lysis of TrampC2 cells was clearly above background when mice had been immunized with MS PTL/ CpG + MS polyl:C, but no lysis over background was observed when effector splenocytes were derived from mice vaccinated with encap sulated adjuvants alone or from mice vaccinated with soluble com pounds. The fibrosarcoma cell line MC57 served as a negative control for the unspecific activation of cytolytic splenocytes (Fig. 5, lower panel).

3.6. Successful immunotherapy of prostate carcinoma in TRAMP mice using PLGA MS containing TRAMP tumor lysate and CpG oligonucleotide

Based on the strong cytolytic T lymphocyte responses obtained in TRAMP mice, we performed a long term experiment of immunotherapy against PCa in TRAMP mice. At the age of 10 weeks, large groups of TRAMP mice were treated with empty MS (empty MS; $n=47$ ), a mix ture of the separately encapsulated TLR ligands CpG and polyI:C (MS CpG + MS polyI:C; $n=29$ ), or MS TrampC2 lysate/CpG in combination with MS polyI:C (MS TPL/CpG + MS polyI:C; $n=41$ ). Subsequently, 

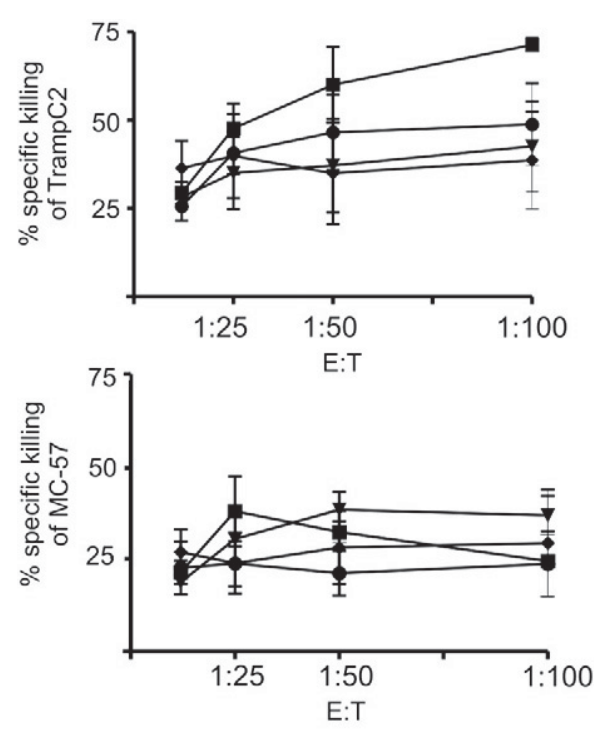

- MS PTL/CpG + MS polylC

$\rightarrow$ soluble PTL + CpG + polyl:C

$\rightarrow-M S C p G+M S$ polyl:C

$\rightarrow-$ empty MS

Fig. 5. Ex vivo cytotoxicity of mouse splenocytes after immunization with PLGA-MS containing TRAMP mouse derived prostate tumor lysate. Groups of mice $(n=8)$ were immunized twice in an interval of two weeks with a mixture of $5 \mathrm{mg}$ MS PTL/CpG and $5 \mathrm{mg}$ MS polyI:C (MS PTL/CpG + MS polyI:C, squares), $5 \mathrm{mg}$ of each of the encapsulated TLR ligands alone (MS CpG + MS polyI:C, triangle), $5 \mathrm{mg}$ of empty MS (empty MS, diamonds), or respective amounts of soluble components (soluble PTL $+\mathrm{CpG}+$ polyl:C, circles). Six days after the second immunization, mice were sacrificed and splenocytes were analyzed for ex vivo cytotoxicity in a DELFIA ${ }^{\circ}$ EuTDA cytotoxicity assay against TrampC2 (upper graph) or MC57 (lower graph) target cell lines.

mice were immunized three more times at biweekly intervals according to the same scheme. At the age of 24 weeks, mice were sacrificed and analyzed by small animal MRI for prostate tumor volume. As Fig. 6 shows, the tumor volume in the group treated with MS containing TRAMP lysate and CpG (MS PTL/CpG) and MS polyI:C was significantly lower than in animals treated with empty MS $\left({ }^{* *} p=0,0036\right)$. Large tumor volumes were almost completely eradicated in the mice treated with MS PTL/CpG + MS polyI:C, whereas mice that were treated with empty MS, showed tumor volumes of up to $10800 \mu \mathrm{l}$. Interestingly, mice treated with the encapsulated TLR ligands $\mathrm{CpG}$ and polyI:C in ab sence of antigen showed a significantly reduced tumor burden at the age of 22 weeks compared to the control group $\left({ }^{*} p=0.0381\right)$, but the therapeutic efficacy was significantly lower compared to immunother apy with PLGA MS that contained tumor lysate $\left({ }^{*} p=0.0338\right)$.

\section{Discussion}

The use of tumor lysates for immunotherapy of cancer has been pursued for a long time, but the clinical efficacy of such approaches has remained low. In this study we have tested the efficacy of micro encapsulating PCa lysates from TRAMP mice together with the TLR9 li gand $\mathrm{CPG}$ oligonucleotide into PLGA MS for the generation of cytolytic $\mathrm{T}$ cell responses and protection of male TRAMP mice from tumor growth. In these proof of principle experiments we used a protocol that we have previously optimized with transplantable model tumors, which is based on the co injection of a mixture of PLGA MS containing antigen and CPG oligo and PLGA MS containing the TLR 3 ligand polyI: $\mathrm{C}[8,16]$. We show that responses of IFN $\gamma$ and granzyme B producing $\mathrm{T}$ cells against TRAMP antigens can be elicited not only in syngeneic C57BL/6 mice, but also in 10 week old TRAMP mice (Fig. 3), which spontaneously develop autochthonous prostate tumors following the onset of puberty [31], and which have proven to be quite resistant to dendritic cell based immunotherapy [24,25]. The TRAMP tumor specif ic T cell responses were detected directly ex vivo without prior restimulation in vitro indicating that they were quite robust. Moreover, the $\mathrm{T}$ cells expressed IFN $\gamma$ and granzyme B, i.e., an important effector cytokine and protease, and were competent to lyse cells.

Vaccination of 10 week old TRAMP mice with PLGA bearing TRAMP lysate and $\mathrm{CpG}$ oligo was capable to reduce strongly the tumor volume as monitored with magnetic resonance imaging on week 24 , when
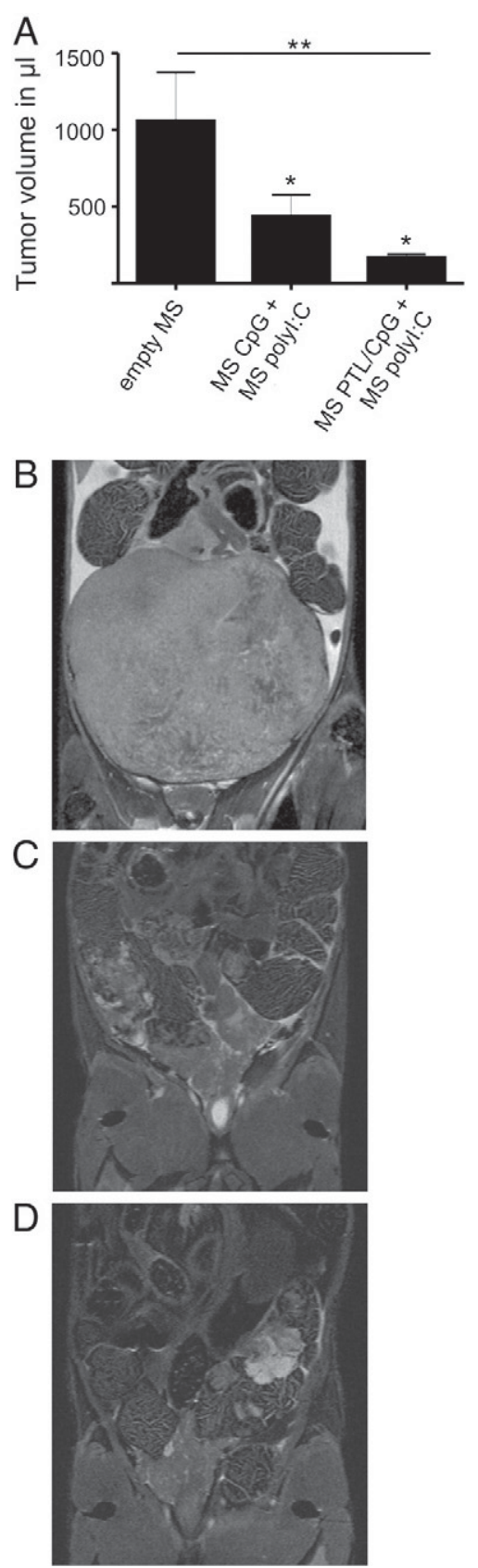

Fig. 6. Immunotherapy of TRAMP mice using TRAMP prostate tumor lysate coencapsulated into PLGA-MS with CpG oligonucleotides, and MS polyI:C. Male TRAMP mice, at the age of ten weeks, were either immunized with $5 \mathrm{mg}$ MS containing TRAMP prostate tumor lysate (PTL) and co-encapsulated CpG and $5 \mathrm{mg}$ MS containing polyI:C (MS PTL/CpG + MS polyI:C; $n=41)$, or empty MS $(n=47)$ as control, or a mixture of MS CpG and MS polyI:C $(n=29)$. Immunizations were repeated three more times at intervals of two weeks. (A) Mice were sacrificed at the age of 24 weeks and subsequently analyzed by magnetic resonance imaging (MRI) for prostate tumor mass, given in $\mu$ l. Differences between groups were assessed by an unpaired Student's $t$-test with Welch's correction. Representative MRI images are shown for each group: (B) Mice treated with empty MS, (C) mice vaccinated with MS CpG + MS polyI:C, or (D) mice vaccinated with MS PTL/CpG + MS polyI:C. 
almost all TRAMP mice already bear large adenocarcinomas and metas tases [31]. The measurement of PCa tissue in the genitourinary tract with small animal MRI as performed in this study has been extensively validated by histological assessment in a previous study from our labo ratory and was shown to be reliable [32]. The outcome of our PLGA MS based immunotherapy of TRAMP mice (Fig. 6) compares favorably with other immunotherapeutic approaches in this animal model; such other immunotherapeutic procedures are, for example, the repet itive combined treatment with DNA vaccine and modified vaccinia virus Ankara expressing the two PCa antigens prostate stem cell anti gen (PSCA) and six transmembrane epithelial antigen of the prostate 1 (STEAP1) [33], or the combined therapy with adoptive transfer of tumor specific cytotoxic $T$ cells and peptide pulsed dendritic cells [25]. Only a recent study employing an optimized DNA vaccine that was electroporated into muscles of TRAMP mice achieved a similar re duction of tumor volume as obtained with the PLGA MS based vaccina tion as investigated here [32].

Most antigens proposed for the use in immunotherapy against PCa are self antigens expressed more or less specifically in the prostate [34]. Whether such prostate specific antigens are appro priate tumor antigens must be questioned. Vaccines employing such antigens have to overcome the mechanisms of thymic and pe ripheral tolerance induction, which is not easily achieved. More promising may be the vaccination against mutated self antigens of the prostate such as the immunodominant SPAS 1 antigen re cently identified in the TRAMP C2 cell line derived from a TRAMP tumor [35]. However, since mutated self antigens vary from tumor to tumor, it seems reasonable to vaccinate against the anti gens derived from the tumor itself. This advantage could be exploited by microencapsulation into PLGA MS of lysates from PCa derived from tumor tissue of the patient to be treated. Primary tumor tissue from radical prostatectomy can be cryopreserved and, in case of a relapse, be rapidly lysed, microencapsulated, and used for immunotherapy as a feasible and convenient individualized therapy. Such very practical approach also has the obvious advan tage that it can be applied irrespective of whether certain MHC al leles are expressed by the patient, which is a prerequisite for peptide based vaccination [36]. One might be concerned that auto immunity may arise as a consequence of PLGA MS mediated vacci nation with autologous tumor lysate, but other procedures using tumor lysates have not resulted in such major adverse reactions. Similarly, our long term experiments with TRAMP mice (Fig. 4) have not evidenced any obvious health problems of the vaccinated mice. It appears that the tolerizing force of the thymus and periph ery is strong enough to prevent autoimmunity, and $\mathrm{T}$ cell response may therefore focus on tumor specific antigens like mutated self antigens.

PLGA MS manufactured by spray drying have many advantages as antigen delivery systems: (i) they are rapidly and efficiently taken up by immature dendritic cells in vitro [9] and in vivo [37] which is due to their optimal size in the low $\mu \mathrm{m}$ range and their sur face charge; (ii) they feed encapsulated antigen efficiently into the presentation pathways of MHC class I and II molecules [12]; (iii) and they suit co encapsulation of antigens and TLR ligands in the same particle, which is important for an optimal anti tumor re sponse $[8,15,16]$. Moreover, their slow release kinetic offers a depot effect (Fig. 3D). However, one disadvantage of PLGA MS as carrier system for protein antigens is the need for relatively impor tant quantities of protein for encapsulation, i.e. typically 5 to $50 \mathrm{mg}$ of protein per batch to obtain a sufficient amount of microspheres for product characterization and immunization of animals to elicit strong and long lasting $\mathrm{T}$ cell responses. Such amounts of highly pu rified recombinant antigens may not be readily available, whereas small pieces of tumor tissue for lysis or of $10^{8}$ tumor cells suffice to prepare a batch of PLGA MS. While recombinant proteins tend to be relatively unstable for proper microencapsulation and release, the microencapsulation of tumor lysates from the three different tu mors EG7 (Fig. 2), Vf10 (Fig. 1), and TRAMP (Figs. 3 and 4) did not represent any issue with respect to batch to batch reproducibility regarding protein and $\mathrm{CpG}$ oligonucleotide release and the efficien cy of $\mathrm{T}$ cell stimulation or the outcome of tumor immunotherapy.

A prerequisite for the clinical application of PLGA MS is product sterility, which can be achieved by aseptic processing or terminal product sterilization. Previously, it has been shown that sterilization of PLGA MS by $\gamma$ irradiation is feasible, simple, and readily applicable, and does not necessarily hamper the release of encapsulated proteins and drugs [38 40]. We tested whether the T cell response to PLGA MS containing ovalbumin or tumor lysate was negatively affected by $\gamma$ irradiation of MS with $25 \mathrm{kGy}$, which was not the case.

\section{Conclusions}

We have shown in a PCa mouse model, which very closely reflects the human disease, that immunotherapy with PLGA MS containing autologous tumor lysates and TLR ligand is feasible, reproducible, and effective. As PLGA is a material approved for clinical application, as resected autologous PCa tissue is often available, and as steriliza tion by $\gamma$ irradiation is applicable, this straight forward approach de serves to be tested in a phase I clinical trial.

Supplementary data to this article can be found online at http:// dx.doi.org/10.1016/j.jconrel.2012.06.015.

\section{Conflict of interest statement}

The authors declare that they have no conflict of interest.

\section{Acknowledgements}

We thank Dr. Wolfram Osen for the EG7 cell line, Dr. Kenneth Rock for the plasmid encoding for ovalbumin, Dr. Michael Basler for scientific and technical advice, and Dr. Bruno Gander for techni cal help and for proofreading the manuscript. This study was supported by a grant from Deutsche Krebshilfe (grant no. 107943) to M.G.

\section{References}

[1] N. Breslow, C.W. Chan, G. Dhom, R.A. Drury, L.M. Franks, B. Gellei, Y.S. Lee, S. Lundberg, B. Sparke, N.H. Sternby, H. Tulinius, Latent carcinoma of prostate at autopsy in seven areas. The International Agency for Research on Cancer, Lyons, France, Int. J. Cancer 20 (1977) 680-688.

[2] M. Basler, M. Groettrup, Advances in prostate cancer immunotherapies, Drugs Aging 24 (2007) 197-221.

[3] M.S. Mitchell, J. Kan-Mitchell, R.A. Kempf, W. Harel, H.Y. Shau, S. Lind, Active specific immunotherapy for melanoma: phase I trial of allogeneic lysates and a novel adjuvant, Cancer Res. 48 (1988) 5883-5893.

[4] J.A. Sosman, V.K. Sondak, Melacine: an allogeneic melanoma tumor cell lysate vaccine, Expert Rev. Vaccines 2 (2003) 353-368.

[5] V.K. Sondak, J.A. Sosman, Results of clinical trials with an allogenic melanoma tumor cell lysate vaccine: melacine, Semin. Cancer Biol. 13 (2003) 409-415.

[6] J. Freund, The effect of paraffin oil and mycobacteria on antibody formation and sensitization; a review, Am. J. Clin. Pathol. 21 (1951) 645-656.

[7] D.E. Speiser, D. Lienard, N. Rufer, V. Rubio-Godoy, D. Rimoldi, F. Lejeune, A.M. Krieg, J.C. Cerottini, P. Romero, Rapid and strong human CD8+ T cell responses to vaccination with peptide, IFA, and CpG oligodeoxynucleotide 7909, J. Clin. Invest. 115 (2005) 739-746.

[8] M. Mueller, E. Schlosser, B. Gander, M. Groettrup, Tumor eradication by immunotherapy with biodegradable PLGA microspheres-an alternative to incomplete Freund's adjuvant, Int. J. Cancer 129 (2011) 407-416.

[9] Y. Waeckerle-Men, E. Scandella, E.U. Allmen, B. Ludewig, S. Gillessen, H.P. Merkle, B. Gander, M. Groettrup, Phenotype and functional analysis of human monocytederived dendritic cells loaded with biodegradable poly(lactide-co-glycolide) microspheres for immunotherapy, J. Immunol. Methods 287 (2004) 109-124.

[10] R. Audran, K. Peter, J. Dannull, Y. Men, M. Groettrup, B. Gander, G. Corradin, Encapsulation of peptides prolongs their presentation to cytotoxic $\mathrm{T}$ cells by antigen presenting cells in vitro, Vaccine 21 (2003) 1250-1255.

[11] K.D. Newman, P. Elamanchili, G.S. Kwon, J. Samuel, Uptake of poly(D,L-lactic-co-glycolic acid) microspheres by antigen-presenting cells in vivo, J. Biomed. Mater. Res. 60 (2002) 480-486. 
[12] Y. WaeckerleMen, E. UetzvonAllmen, B. Gander, E. Scandella, E. Schlosser, G. Schmidtke, H.R. Merkle, M. Groettrup, Encapsulation of proteins and peptides into biodegradable poly(D, L-lactide-co-glycolide) microspheres prolongs and enhances antigen presentation by human dendritic cells, Vaccine 24 (2006) 1847-1857.

[13] Z. Shen, G. Reznikoff, G. Dranoff, K.L. Rock, Cloned dendritic cells can present exogenous antigens on both MHC class I and class II molecules, J. Immunol. 158 (1997) 2723-2730

[14] V. Manolova, A. Flace, M. Bauer, K. Schwarz, P. Saudan, M.F. Bachmann, Nanoparticles target distinct dendritic cell populations according to their size, Eur. J. Immunol. 38 (2008) 1404-1413.

[15] A. Heit, F. Schmitz, T. Haas, D.H. Busch, H. Wagner, Antigen co-encapsulated with adjuvants efficiently drive protective T cell immunity, Eur. J. Immunol. 37 (2007) 2063-2074.

[16] E. Schlosser, M. Mueller, S. Fischer, S. Basta, D.H. Busch, B. Gander, M. Groettrup, TLR ligands and antigen need to be coencapsulated into the same biodegradable microsphere for the generation of potent cytotoxic T lymphocyte responses, Vaccine 26 (2008) 1626-1637.

[17] C.M. Solbrig, J.K. Saucier-Sawyer, V. Cody, W.M. Saltzman, D.J. Hanlon, Polyme nanoparticles for immunotherapy from encapsulated tumor-associated antigens and whole tumor cells, Mol. Pharm. 4 (2007) 47-57.

[18] S. Hamdy, O. Molavi, Z. Ma, A. Haddadi, A. Alshamsan, Z. Gobti, S. Elhasi, J. Samuel A. Lavasanifar, Co-delivery of cancer-associated antigen and Toll-like receptor 4 ligand in PLGA nanoparticles induces potent CD8+ T cell-mediated anti-tumor immunity, Vaccine 26 (2008) 5046-5057.

[19] R. Goforth, A.K. Salem, X. Zhu, S. Miles, X.Q. Zhang, J.H. Lee, A.D. Sandler, Immune stimulatory antigen loaded particles combined with depletion of regulatory T-cells induce potent tumor specific immunity in a mouse model of melanoma, Cancer Immunol. Immunother. 58 (2009) 517-530.

[20] G. Napolitani, A. Rinaldi, F. Bertoni, F. Sallusto, A. Lanzavecchia, Selected Toll-like receptor agonist combinations synergistically trigger a T helper type 1-polarizing program in dendritic cells, Nat. Immunol. 6 (2005) 769-776.

[21] N.M. Greenberg, F. DeMayo, M.J. Finegold, D. Medina, W.D. Tilley, J.O. Aspinall, G.R. Cunha, A.A. Donjacour, R.J. Matusik, J.M. Rosen, Prostate cancer in a transgenic mouse, Proc. Natl. Acad. Sci. U. S. A. 92 (1995) 3439-3443.

[22] N. Wernert, E. Bieroff, A. Hugel, Pathological aspects of prostate cancer and benign nodular hyperplasia, Anticancer Res. 17 (1997) 2907-2910.

[23] S.B. Shappell, G.V. Thomas, R.L. Roberts, R. Herbert, M.M. Ittmann, M.A. Rubin, P.A. Humphrey, J.P. Sundberg, N. Rozengurt, R. Barrios, J.M. Ward, R.D. Cardiff, Prostate pathology of genetically engineered mice: definitions and classification. The consensus report from the Bar Harbor meeting of the Mouse Models of Human Cancer Consortium Prostate Pathology Committee, Cancer Res. 64 (2004) 2270-2305.

[24] E. Degl'Innocenti, M. Grioni, A. Boni, A. Camporeale, M.T. Bertilaccio, M. Freschi, A. Monno, C. Arcelloni, N.M. Greenberg, M. Bellone, Peripheral T cell tolerance occurs early during spontaneous prostate cancer development and can be rescued by dendritic cell immunization, Eur. J. Immunol. 35 (2005) 66-75.

[25] M.J. Anderson, K. Shafer-Weaver, N.M. Greenberg, A.A. Hurwitz, Tolerization of tumor-specific $\mathrm{T}$ cells despite efficient initial priming in a primary murine model of prostate cancer, J. Immunol. 178 (2007) 1268-1276.
[26] K.A. Shafer-Weaver, S.K. Watkins, M.J. Anderson, L.J. Draper, A. Malyguine, W.G. Alvord, N.M. Greenberg, A.A. Hurwitz, Immunity to murine prostatic tumors: continuous provision of T-cell help prevents CD8 T-cell tolerance and activates tumor-infiltrating dendritic cells, Cancer Res. 69 (2009) 6256-6264.

[27] P. Johansen, Y. Men, H.P. Merkle, B. Gander, Revisiting PLA/PLGA microspheres: an analysis of their potential in parenteral vaccination, Eur. J. Pharm. Biopharm. 50 (2000) 129-146.

[28] B.A. Foster, J.R. Gingrich, E.D. Kwon, C. Madias, N.M. Greenberg, Characterization of prostatic epithelial cell lines derived from transgenic adenocarcinoma of the mouse prostate (TRAMP) model, Cancer Res. 57 (1997) 3325-3330.

[29] L.J. Shen, K.L. Rock, Cellular protein is the source of cross-priming antigen in vivo, Proc. Natl. Acad. Sci. U. S. A. 101 (2004) 3035-3040.

[30] K. Blomberg, R. Hautala, J. Lovgren, V.M. Mukkala, C. Lindqvist, K. Akerman, Time-resolved fluorometric assay for natural killer activity using target cells labelled with a fluorescence enhancing ligand, J. Immunol. Methods 193 (1996) 199-206.

[31] J.R. Gingrich, R.J. Barrios, R.A. Morton, B.F. Boyce, F.J. DeMayo, M.J. Finegold, R. Angelopoulou, J.M. Rosen, N.M. Greenberg, Metastatic prostate cancer in a transgenic mouse, Cancer Res. 56 (1996) 4096-4102.

[32] E. Spies, W. Reichardt, G. Alvarez, M. Groettrup, P. Ohlschlager, An artificial PAP gene breaks self-tolerance and promotes tumor regression in the TRAMP model for prostate carcinoma, Mol. Ther. 20 (3) (2011) 555-564.

[33] M. Krupa, M. Canamero, C.E. Gomez, J.L. Najera, J. Gil, M. Esteban, Immunization with recombinant DNA and modified vaccinia virus Ankara (MVA) vectors delivering PSCA and STEAP1 antigens inhibits prostate cancer progression, Vaccine 29 (2011) 1504-1513.

[34] A.C. Cunha, B. Weigle, A. Kiessling, M. Bachmann, E.P. Rieber, Tissue specificity of prostate specific antigens: comparative analysis of transcript levels in prostate and non-prostatic tissues, Cancer Lett. 236 (2006) 229-238.

[35] M. Fasso, R. Waitz, Y. Hou, T. Rim, N.M. Greenberg, N. Shastri, L. Fong J.P. Allison, SPAS-1 (stimulator of prostatic adenocarcinoma-specific T cells)/SH3GLB2: a prostate tumor antigen identified by CTLA-4 blockade, Proc. Natl. Acad. Sci. U. S. A. 105 (2008) 3509-3514.

[36] Y. Waeckerle-Men, E. Uetz-von Allmen, M. Fopp, R. von Moos, C. Boehme, H.P. Schmid, D. Ackermann, T. Cerny, B. Ludewig, M. Groettrup, S. Gillessen, Dendritic cell-based multi-epitope immuntherapy of hormone-refractory prostate carcinoma, Cancer Immunol. Immunother. 55 (2006) 1524-1533.

[37] C. Schliehe, C. Redaelli, S. Engelhardt, M. Fehlings, M. Mueller, N. van Rooijen, M. Thiry, K. Hildner, H. Weller, M. Groettrup, CD8 dendritic cells and macrophages cross-present PLGA particle-encapsulated antigen in vivo, J. Immunol. 187 (2011) 2112-2121.

[38] R. Herrero-Vanrell, L. Ramirez, A. Fernandez-Carballido, M.F. Refojo, Biodegradable PLGA microspheres loaded with ganciclovir for intraocular administration. Encapsulation technique, in vitro release profiles, and sterilization process, Pharm. Res. 17 (2000) 1323-1328.

[39] D. Mohanan, B. Gander, T.M. Kundig, P. Johansen, Encapsulation of antigen in poly(d,l-lactide-co-glycolide) microspheres protects from harmful effects of gamma-irradiation as assessed in mice, Eur. J. Pharm. Biopharm. 80 (2) (2011) 274-281.

[40] Y. Yang, Y. Gao, X. Mei, Effects of gamma-irradiation on PLGA microspheres loaded with thienorphine, Pharmazie 66 (2011) 694-697. 